Enhanced dynamic annealing and optical activation of Eu implanted a-plane GaN

This article has been downloaded from IOPscience. Please scroll down to see the full text article.

2012 EPL 9768004

(http://iopscience.iop.org/0295-5075/97/6/68004)

View the table of contents for this issue, or go to the journal homepage for more

Download details:

IP Address: 147.96.14.16

The article was downloaded on 20/02/2013 at 17:28

Please note that terms and conditions apply. 


\title{
Enhanced dynamic annealing and optical activation of Eu implanted a-plane GaN
}

\author{
N. Catarino ${ }^{1}$, E. Nogales ${ }^{2}$, N. Franco ${ }^{1,3}$, V. Darakchieva ${ }^{1,3(a)}$, S. M. C. Miranda ${ }^{1}$, B. Méndez $^{2}$, \\ E. Alves ${ }^{1,3}$, J. G. Marques ${ }^{1,3}$ and K. Lorenz ${ }^{1,3(b)}$ \\ ${ }^{1}$ Instituto Tecnológico e Nuclear/IST - Estrada Nacional 10, 2686-953 Sacavém, Portugal, EU \\ ${ }^{2}$ Departamento de Fúsica de Materiales, Universidad Complutense - 28040 Madrid, Spain, EU \\ ${ }^{3}$ Centro de Física Nuclear da Universidade de Lisboa - Av. Prof. Gama Pinto, 1649-003 Lisboa, Portugal, EU
}

received 5 January 2012; accepted in final form 15 February 2012

published online 20 March 2012

PACS 81.05.Ea - III-V semiconductors

PACS 61.72.U- - Doping and impurity implantation

PACS 78.60. Hk - Cathodoluminescence, ionoluminescence

\begin{abstract}
The implantation damage build-up and optical activation of $a$-plane and $c$-plane GaN epitaxial films were compared upon $300 \mathrm{keV}$ Eu implantation at room temperature. The implantation defects cause an expansion of the lattice normal to the surface, i.e. along the $a$-direction in $a$-plane and along the $c$-direction in $c$-plane GaN. The defect profile is bimodal with a pronounced surface damage peak and a second damage peak deeper in the bulk of the samples in both cases. For both surface orientations, the bulk damage saturates for high fluences. Interestingly, the saturation level for $a$-plane $\mathrm{GaN}$ is nearly three times lower than that for $c$-plane material suggesting very efficient dynamic annealing and strong resistance to radiation. $a$-plane GaN also shows superior damage recovery during post-implant annealing compared to $c$-plane $\mathrm{GaN}$. For the lowest fluence, damage in $a$-plane GaN was fully removed and strong Eu-related red luminescence is observed. Although some residual damage remained after annealing for higher fluences as well as in all $c$-plane samples, optical activation was achieved in all samples revealing the red emission lines due to the ${ }^{5} D_{0} \rightarrow{ }^{7} F_{2}$ transition in the $\mathrm{Eu}^{3+}$ ion. The presented results demonstrate a great promise for the use of ion beam processing for $a$-plane GaN based electronic devices as well as for the development of radiation tolerant electronics.
\end{abstract}

Copyright (C) EPLA, 2012

Introduction. - Rare-earth (RE) doped III-nitrides are widely studied with respect to applications in optoelectronics and spintronics [1]. In particular, Eu-doped GaN has emerged as efficient red-light emitter and possible alternative to conventional InGaN-based LEDs which show low efficiencies in the green and red spectral region [2]. GaN:Eu was also reported to exhibit room temperature ferromagnetism [3]. Ion implantation is an attractive technique to incorporate $\mathrm{RE}$ ions with well-defined depth profiles allowing for easy lateral patterning. c-plane GaN, typically grown heteroepitaxially on (0001) sapphire substrates, is considered very resistant to implantation damage due to strong dynamic annealing effects [4]. However, the heavy RE ions create severe lattice damage which could only be efficiently removed by thermal annealing at ultrahigh temperatures

(a) Present address: Department of Physics, Chemistry and Biology, Linköping University - Linköping SE-58183, Sweden, EU.

(b) E-mail: lorenz@itn.pt and nitrogen pressures $[5,6]$. Therefore, it is of utmost importance to keep implantation damage low already during the implantation. Channelled implantation along the $c$-axis and implantation at elevated temperatures proved successful to decrease implantation damage $[7,8]$.

In recent years, growth of GaN along non-polar directions has been investigated due to the possibility of eliminating detrimental polarization effects in nitride LEDs [9]. As a result of this research, high-quality GaN templates grown with the non-polar $a$ - or $m$-plane surface orientations became available. Implantation studies in these novel materials remain scarce. Implantation of transition metals in $a$-plane GaN has been studied for spintronics applications $[10,11]$. However, no detailed structural and optical characterization has been performed to understand the implantation damage build-up in $a$-plane GaN.

In this work we compare the damage build-up behaviour of $c$-plane and $a$-plane GaN upon Eu implantation. It is shown that $a$-plane $\mathrm{GaN}$ is considerably more resistant to radiation damage than $c$-plane material. 
Experimental details. - $c$-plane GaN grown on $c$-plane sapphire and $a$-plane GaN grown on $r$-plane sapphire by hydride vapour phase epitaxy, $\sim 3 \mu \mathrm{m}$ thick, were purchased from TDI (Oxford Instruments). Samples were implanted with $300 \mathrm{keV} \mathrm{Eu}$ ions to fluences ranging from $1 \times 10^{14}$ to $5 \times 10^{15} \mathrm{Eu} / \mathrm{cm}^{2}$. The implantations were carried out at room temperature and along the surface normal of the samples.

Annealing of selected samples was performed for $20 \mathrm{~min}$ at $1000{ }^{\circ} \mathrm{C}$ in a tube furnace under flowing nitrogen. An unimplanted piece of $\mathrm{GaN}$ was placed face to face to the samples as a proximity cap to minimize the out-diffusion of nitrogen.

Structural characterization of the samples was performed by Rutherford backscattering spectrometry and channelling (RBS/C) using a $1 \mathrm{~mm}$ diameter collimated beam of $2 \mathrm{MeV} \mathrm{He}^{+}$ions. The backscattered particles were detected at $140^{\circ}$ with respect to the incoming beam direction using a silicon surface barrier detector. Aligned spectra were acquired along the $\langle 0001\rangle$ and $\langle 11 \overline{2} 0\rangle$ axes for the $c$ - and $a$-plane GaN, respectively. The Eu concentration profiles were determined from the random RBS spectra using the NDF code [12]. To extract the defect profiles from the RBS/C spectra, dechannelling was taken into account using the DICADA code [13] and assuming a Debye temperature of $365 \mathrm{~K}$ [14].

High-resolution X-ray diffraction (XRD) curves and reciprocal space maps (RSMs) were acquired using monochromated $\mathrm{CuK}_{\alpha 1}$ radiation on a D8Discover system from Bruker-AXS using a Göbel mirror and an asymmetric 2-bounce $\mathrm{Ge}(220)$ monochromator in the primary beam and a $1 \mathrm{~mm}$ slit in front of a scintillation detector in the secondary beam.

Cathodoluminescence (CL) was shown to be a powerful technique for the characterization of $a$-plane nitrides [15] and was used in this study to investigate the optical activation of the Eu ions. The CL measurements were performed at room temperature in a Hitachi S2500 scanning electron microscope using a Hamamatsu PMA-11 charge coupling device camera.

Results and discussion. - Figure 1(a) shows typical random and aligned RBS/C spectra for $a$ - and $c$-plane GaN implanted to a fluence of $2.4 \times 10^{15} \mathrm{Eu} / \mathrm{cm}^{2}$. The $\mathrm{RBS} / \mathrm{C}$ minimum yield, $\chi_{\min }$, is the ratio between the yield in the aligned and the random spectra and serves to quantify the single crystalline quality. The aligned spectra of the as-grown samples are also shown in fig. 1(a) and evidence the good crystal quality of both materials with minimum yields of $\sim 2 \%$. A slightly better crystal quality of the as-grown material is found for the $c$-plane GaN. The Eu profiles (fig. 1(b)) are identical for both materials showing the maximum Eu concentration of $\sim 0.5$ at. $\%$ at a depth of $\sim 50 \mathrm{~nm}$.

Both materials show a bimodal distribution of implantation defects with one defect peak at the surface and another one deeper inside the sample (respectively marked
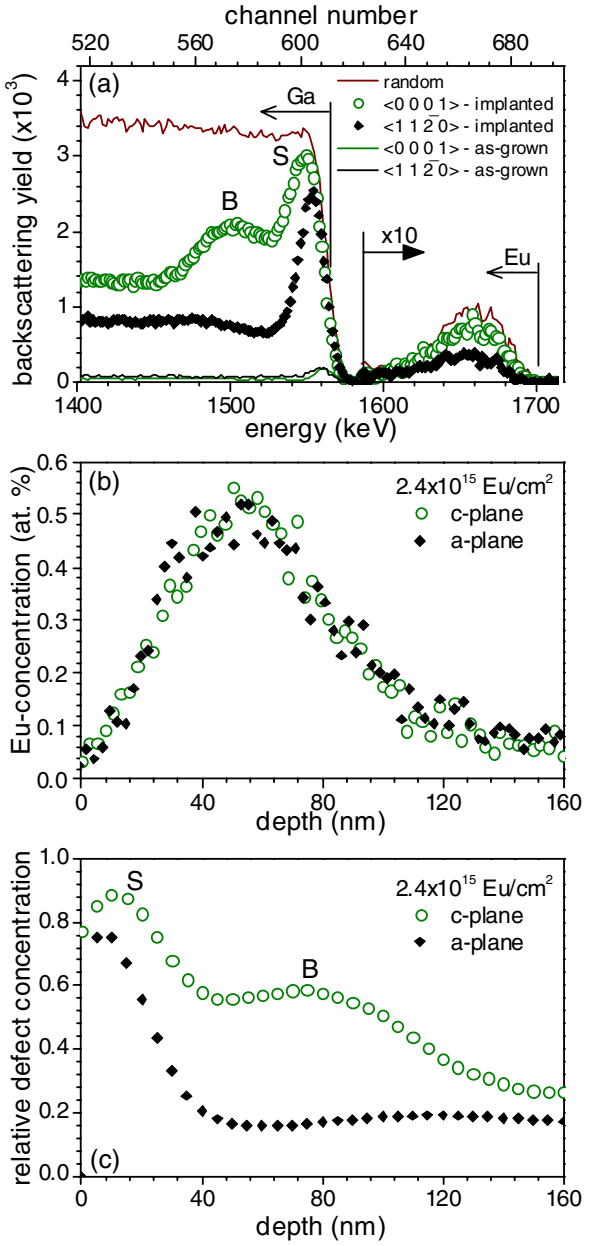

Fig. 1: (Colour on-line) (a) RBS/C random and aligned spectra of $a$ - and $c$-plane GaN implanted with $2.4 \times 10^{15} \mathrm{Eu} / \mathrm{cm}^{2}$. Aligned spectra of the as-grown samples are shown for comparison. (b) The Eu profiles extracted from the spectra in (a). (c) The defect profiles in the Ga sublattice extracted from the spectra in (a).

$\mathrm{S}$ and $\mathrm{B}$ in fig. 1). The pronounced surface damage is a particularity for room temperature implantation in GaN and was attributed to the trapping of diffusing point defects $[4,16,17]$. In $c$-plane GaN, the surface peak was shown to consist of randomly oriented nanocrystallites for identical implantation parameters [18].

The defect profiles extracted from the RBS/C spectra of fig. 1(a) are shown in fig. 1(c). A pronounced difference is seen, in particular in the bulk where $a$-plane material shows a considerably lower damage level. The identical Eu profiles (fig. 1(b)) show that ion channelling effects are similar for both orientations and cannot be the reason for the different damage behaviour.

Figure 2 presents the relative damage level in the maximum of the bulk damage peak as a function of the fluence directly after the implantation and after annealing. Note that the damage can only be measured for the Ga sublattice, due to the low $\mathrm{N}$ backscattering yield. The 


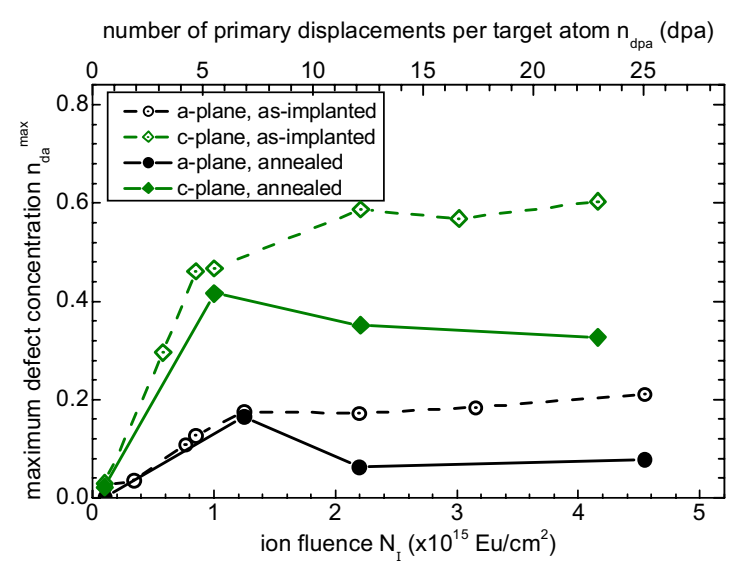

Fig. 2: (Colour on-line) Maximum relative defect concentrations in the Ga-sublattice in the bulk of $c$-plane and $a$-plane GaN before and after annealing as a function of the implantation fluence (also converted to displacements per atom).

fluence was also converted into number of displacements per atom $\left(n_{\text {dpa }}\right)$ using $n_{\text {dpa }}=N_{\text {displ }} \cdot N_{\mathrm{I}} / N_{0}$, where $N_{\text {displ }}$ is the number of $\mathrm{Ga}$ displacements per ion and unit depth at the maximum of the damage profile, $N_{\mathrm{I}}$ is the ion fluence and $N_{0}=4.385 \times 10^{22} \mathrm{Ga} / \mathrm{cm}^{3}$ the atomic $\mathrm{Ga}$ density. $N_{\text {displ }}$ is calculated using SRIM 2008 [19] with values for the displacement energies, $E_{\mathrm{D}}$, for $\mathrm{Ga}$ and $\mathrm{N}$ of $41 \mathrm{eV}$ [20]. Although molecular-dynamic calculations reveal slightly different values of $E_{\mathrm{D}}$ for different crystal directions [21], in the dense collision cascade caused by heavy ions these differences will have minor effects on the overall damage.

The single crystalline nature of all samples is preserved even for $n_{\text {dpa }}$ as high as 25. For $c$-plane material, the bulk damage first increases with the fluence and then saturates for fluences above $1 \times 10^{15} \mathrm{Eu} / \mathrm{cm}^{2}$ at defect levels around 0.5-0.6 (fig. 2), which agrees excellently with previous results for $300 \mathrm{keV} \mathrm{RE}$ implantation at room temperature [8]. We observe a similar behaviour for the $a$-plane material but with a considerably lower saturation level of $\sim 0.2$ (fig. 2). Similar results are found for random implantations where the beam direction was tilted away from the surface normal by $10^{\circ}$ (not shown). This, in addition to the similar Eu profiles shown in fig. 1(b), shows that channelling effects do not play a major role in the observed differences in damage accumulation.

The saturation of defect concentration is usually explained by efficient dynamic annealing leading to the recombination of created vacancies and interstitials or to a diffusion of point defects towards deeper regions or towards the surface during the implantation [4]. The lower saturation level in the $a$-plane GaN suggests a strong enhancement of such diffusion processes in this case. The diffusion of defects as well as the annihilation of defects at the sample surface are likely to depend strongly on the orientation of the crystal. For instance, strong anisotropies in dynamic annealing efficiency for crystals

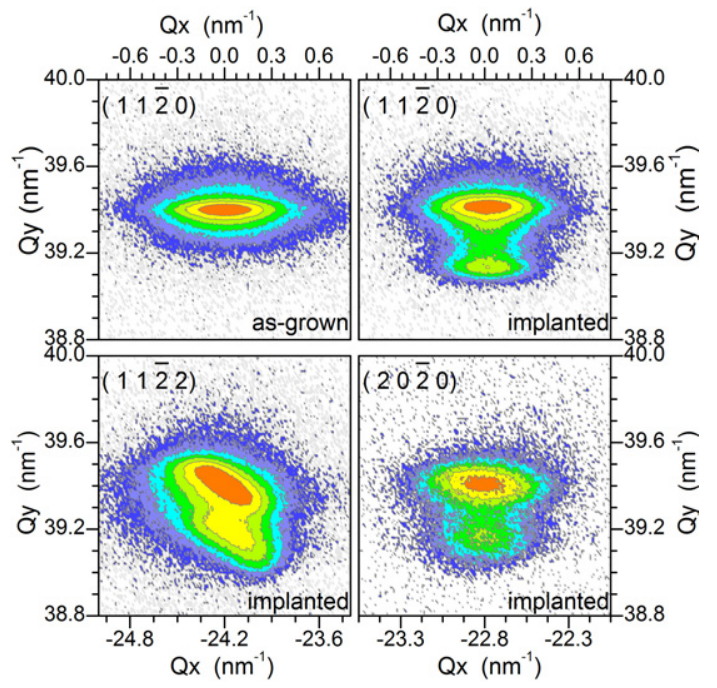

Fig. 3: (Colour on-line) XRD RSMs of an as-grown $a$-plane GaN sample and after implantation of $1 \times 10^{14} \mathrm{Eu} / \mathrm{cm}^{2}$.

with different surface orientation were also reported for $\mathrm{MgO}$ crystals [22]. Also a different microscopic nature of defects formed during the implantation can influence the damage accumulation seen by RBS. In c-plane material, basal stacking faults and point defect clusters are the dominant defects caused by the implantation $[18,23]$. Such extended defects may trap diffusing point defects and therefore slow down dynamic annealing processes. This would suggest a lower production rate of extended defects in $a$-plane material; however, the microscopic nature of the implantation damage in $a$-plane GaN needs to be investigated to confirm this hypothesis.

Eu typically incorporates at near-substitutional Ga sites in c-plane GaN [6]. The decreased backscattering yield from $\mathrm{Eu}$ in the aligned spectrum for the $a$-plane GaN (fig. 1(a)) indicates that Eu is incorporated on substitutional Ga sites as in $c$-plane GaN. For an exact lattice site location study, however, it is necessary to measure full angular channelling scans along several crystal directions. Interestingly, the lower backscattering yield for $\mathrm{Eu}$ in the aligned $a$-plane sample compared to that of the $c$-plane sample (see fig. 1) suggests a higher substitutional fraction for the former. This is in agreement with previous measurements in c-plane GaN showing decreasing substitutional fractions for increasing defect levels [8].

We find that implantation damage in the $c$-plane epitaxial GaN layers leads to a strong expansion of the c-lattice parameter while the $a$-lattice parameter remains constant in agreement with previous works $[7,24,25]$. To investigate the lattice expansion upon implantation in the $a$-plane GaN, we measured XRD RSMs around the $11 \overline{2} 0,11 \overline{2} 2$ and $20 \overline{2} 0$ reciprocal lattice points (fig. 3 ). The implanted layer gives rise to an additional peak in all RSMs at lower $Q y$ positions reflecting an expansion of the lattice along the $[11 \overline{2} 0]$ direction ( $a$-direction). The $Q x$ 


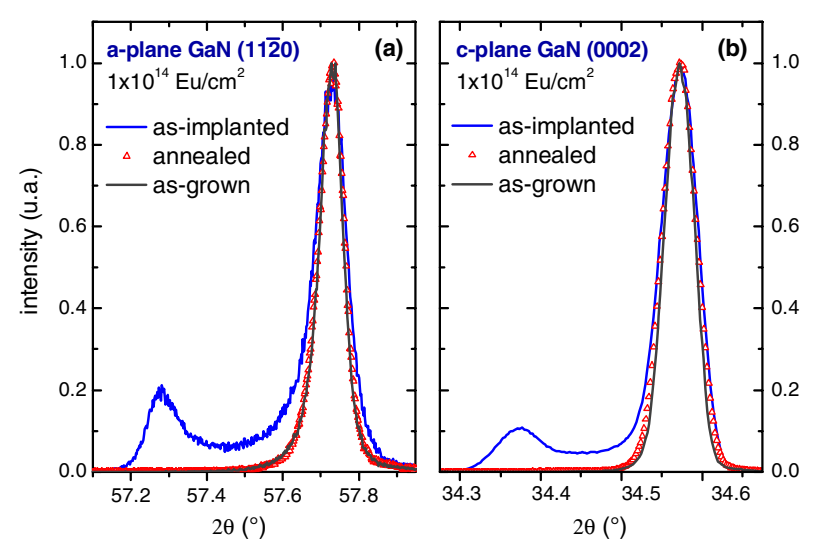

Fig. 4: (Colour on-line) XRD 2 $\theta-\omega$ curves of (a) $a$-plane GaN (11̄20 reflection) and (b) c-plane GaN (0002 reflection) implanted to a fluence of $1 \times 10^{14} \mathrm{Eu} / \mathrm{cm}^{2}$. Curves for the as-grown, the as-implanted and the implanted and annealed samples are compared.

values of the $(11 \overline{2} 2)$ and $(20 \overline{2} 0)$ maps are proportional to the inverse lattice spacing along the $c$ - and $m$-direction, respectively. No deformation of the RSMs are observed along $Q x$ showing that implantation damage in $a$-plane GaN leads to an expansion only along the $a$-direction while the lattice spacings in the perpendicular directions remain unchanged.

Preliminary annealing studies between $800{ }^{\circ} \mathrm{C}$ and $1000^{\circ} \mathrm{C}$ using the perturbed angular correlation technique indicated similar annealing behaviour for $a$ - and c-plane $\mathrm{GaN}$ for very low fluences $\left(<10^{13} \mathrm{Hf} / \mathrm{cm}^{2}\right)[26]$. The best recovery was found for $1000^{\circ} \mathrm{C}$. Previously it was shown for $c$-plane $\mathrm{GaN}$ that the annealing time played a minor role in damage recovery [17]. In the present case, annealing promotes the recovery of the structural defects as seen in the decreasing damage levels for all samples after annealing (fig. 2). However, a full recovery of the minimum yield of the as-grown sample is only achieved for $a$-plane GaN implanted to the lowest fluence of $1 \times 10^{14} \mathrm{Eu} / \mathrm{cm}^{2}$. The good recovery of this sample is also manifested in the XRD $2 \theta-\omega$ curves of the $11 \overline{2} 0$ reflection presented in fig. 4(a). In the as-implanted sample the typical satellite peak at lower angles, due to the expansion of the lattice in the implanted layer, is clearly seen. After annealing this expansion is completely reversed and the XRD curve coincides with that of the as-grown sample. The $c$-plane sample, implanted and annealed in the same conditions as the $a$-plane film, also reveals a good recovery, however, the XRD curve after annealing is slightly broader than that for the as-grown sample (fig. 4(b)) indicating the presence of residual defects in agreement with the RBS/C results.

Hao et al. reported an increased anisotropy of the widths of $11 \overline{2} 0$ rocking curves (RC) after annealing $a$-plane GaN at $1070^{\circ} \mathrm{C}$ which was attributed to the redistribution of dislocations during the annealing; the $\mathrm{RC}$ acquired

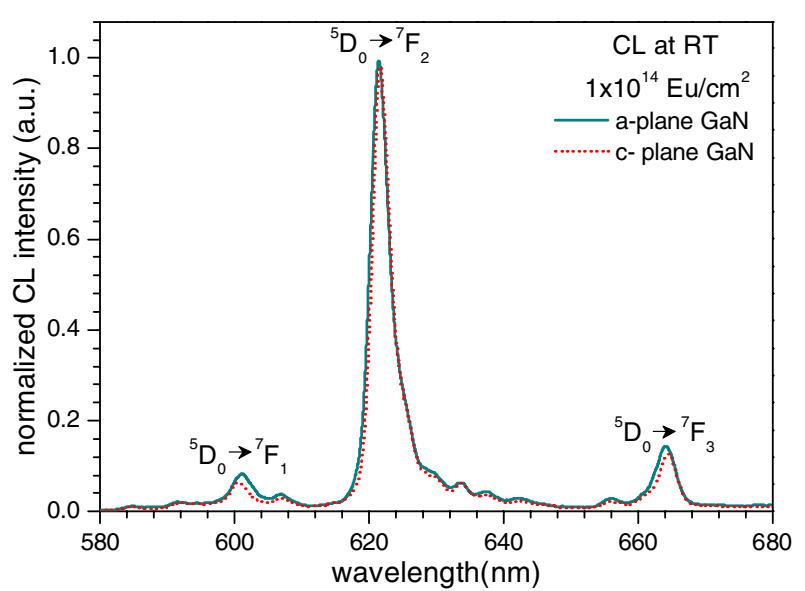

Fig. 5: (Colour on-line) Normalized CL spectra of the $a$-plane $\mathrm{GaN}$ and the $c$-plane GaN implanted to a fluence of $1 \times 10^{14} \mathrm{Eu} / \mathrm{cm}^{2}$ after annealing. The spectral region for the ${ }^{5} D_{0} \rightarrow{ }^{7} F_{2}$ transition is shown.

with the X-ray beam aligned with the [0001] direction were seen to broaden strongly while those taken in the perpendicular direction narrowed [27]. We have extracted $\mathrm{RC}$ from the $11 \overline{2} 0$ RSM taken with the beam parallel and perpendicular to the [0001] direction. All RC broaden slightly after implantation and decrease again after annealing. In contrast to ref. [27], we see a decreased anisotropy after annealing; the $\mathrm{RC}$ width along [0001] decreases from $0.44^{\circ}$ in the as-grown to $0.41^{\circ}$ in the annealed sample while that in the perpendicular direction does not change significantly $\left(\mathrm{FWHM} \sim 0.21^{\circ}\right)$. The difference might be due to the lower annealing temperature which is below typical growth temperatures for GaN. Also the nature and distribution of native defects may be different in our samples.

After annealing, optical activation of the $\mathrm{Eu}^{3+}$ ions is observed for all samples. Figure 5 compares the CL spectra of $a$-plane and $c$-plane GaN implanted to a fluence of $1 \times 10^{14} \mathrm{Eu} / \mathrm{cm}^{2}$. The spectra were normalized in order to compare the spectral shape of the narrow $\mathrm{Eu}^{3+}$ emission lines which correspond to the ${ }^{5} D_{0} \rightarrow{ }^{7} F_{2}$ intra- $4 f$ transitions. The shape is almost identical for both GaN surface orientations indicating that $\mathrm{Eu}$ occupies identical lattice sites in both materials. This is in agreement with the RBS/C results suggesting a preferential incorporation of $\mathrm{Eu}$ in substitutional Gasites. The slight differences may be due to the different strain states of the materials with different orientations. Figure 6 presents the integrated CL intensity for the ${ }^{5} D_{0} \rightarrow{ }^{7} F_{2}$ transitions at $\sim 620 \mathrm{~nm}$. For both materials the CL intensity is seen to increase with the fluence up to $2 \times 10^{15} \mathrm{at} / \mathrm{cm}^{2}$. For the highest fluence a pronounced drop of CL intensity is observed due to the severe lattice damage. For low fluences the $a$-plane GaN reveals brighter CL than $c$-plane material in good agreement with its superior structural quality especially for the sample 


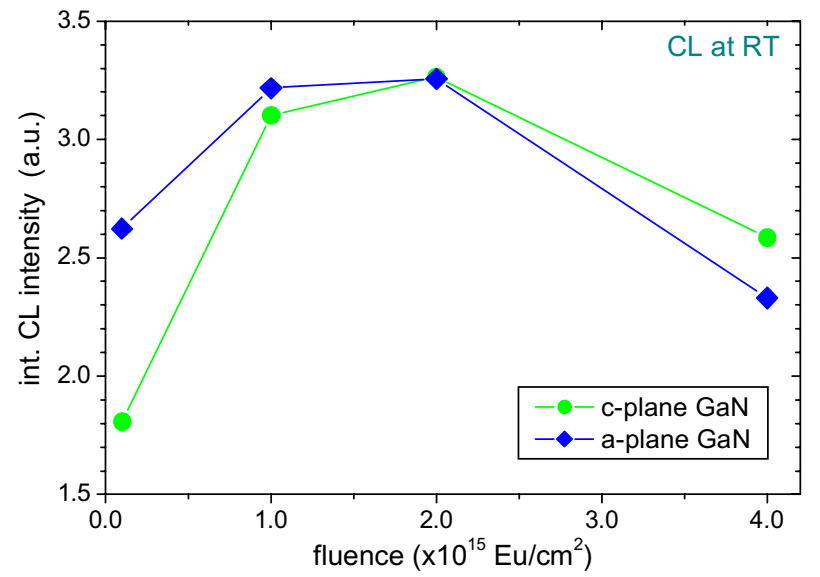

Fig. 6: (Colour on-line) Integrated red CL intensity around $\sim 620 \mathrm{~nm}$ as a function of the fluence for Eu implanted and annealed $a$-plane and $c$-plane GaN.

implanted to $1 \times 10^{14} \mathrm{Eu} / \mathrm{cm}^{2}$ which showed full structural recovery.

Conclusions. - Eu ions have been implanted into $a$-plane and $c$-plane GaN epitaxial films in order to study the build-up of implantation damage for the two different surface orientations at room temperature. Implantation damage leads to an expansion of the lattice perpendicular to the surface while the in-plane lattice parameters remain unchanged, i.e. we observe an expansion along the $a$-direction in $a$-plane material and along the $c$-direction in $c$-plane material. For both orientations the damage profile is bimodal with a damage peak at the surface and another one deeper in the bulk. The bulk damage saturates for fluences above $1 \times 10^{15} \mathrm{Eu} / \mathrm{cm}^{2}$. The saturation defect level in $a$-plane GaN is three times lower than that observed in $c$-plane GaN indicating a different microstructure of the created damage and a strongly enhanced dynamic annealing effect.

Superior annealing characteristics were observed for $a$-plane GaN. The crystal quality of an $a$-plane GaN sample implanted to a fluence of $1 \times 10^{14} \mathrm{Eu} / \mathrm{cm}^{2}$ could be completely recovered while $c$-plane GaN still revealed residual defects after annealing.

Eu was optically activated after annealing in all samples. In good agreement with the superior structural quality, $a$-plane GaN revealed higher CL intensities than $c$-plane GaN for low fluences.

The presented results suggest that $a$-plane GaN may be more suitable for device processing using ion beams than conventional $c$-plane material. The reduced implantation damage and improved annealing characteristics of $a$-plane GaN, shown here for Eu implantation, will also improve doping characteristics after implantation of electrical or magnetic dopants. Furthermore, the enhanced dynamic annealing in $a$-plane GaN suggests that electronics based on $a$-plane GaN may be more radiation resistant than conventional devices based on polar material.

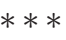

Financial support by FCT Portugal (Ciência 2007, PTDC/CTM/100756/2008) and through the bilateral Spanish-Portuguese project HP-2008-0071 is gratefully acknowledged.

\section{REFERENCES}

[1] O'Donnell K. P. and Dierolf V. (Editors), RareEarth Doped III-Nitrides for Optoelectronic and Spintronic Applications (Spinger, Dordrecht) 2010.

[2] Nishikawa A., Kawasaki T., Furukawa N., Terai Y. and Fujiwara Y., Appl. Phys. Express, 2 (2009) 071004.

[3] Hite J., Thaler G. T., Khanna R., Abernathy C. R., Pearton S. J., Park J. H., Steckl A. J. and Zavada J. M., Appl. Phys. Lett., 89 (2006) 132119.

[4] Kucheyev S. O., Williams J. S. and Pearton S. J., Mater. Sci. Eng. R, 33 (2001) 51.

[5] Roqan I. S., O'Donnell K. P., Martin R. W., Edwards P. R., Song S. F., Vantomme A., Lorenz K., Alves E. and Boćkowski M., Phys. Rev. B, 81 (2010) 085209.

[6] Lorenz K., Alves E., Roqan I. S., O’Donnell K. P., Nishikawa A., Fujiwara Y. and BoćKowski M., Appl. Phys. Lett., 97 (2010) 111911.

[7] Pipeleers B., Hogg S. M. and Vantomme A., J. Appl. Phys., 98 (2005) 123504.

[8] Lorenz K., Barradas N. P., Alves E., Roqan I. S., Nogales E., Martin R. W., O'Donnell K. P., Gloux F. and Ruterana P., J. Phys. D: Appl. Phys., 42 (2009) 165103.

[9] Speck J. S. and Chichibu S. F. (Guest Editors), MRS Bull., 34 (2009) 304.

[10] Sun L., Yan F., Zhang H., Wang J., Zeng Y., Wang G. and Li J., J. Appl. Phys., 106 (2009) 113921.

[11] Sun L., Yan F., GaO H., Zhang H., Zeng Y., Wang G. and Li J., J. Phys. D: Appl. Phys., 41 (2008) 165004.

[12] Barradas N. P., Jeynes C. and Webb R. P., Appl. Phys. Lett., 71 (1997) 291.

[13] Gärtner K., Nucl. Instrum. Methods Phys. Res. B, 227 (2005) 522.

[14] Danilchenko B. A., Paszkiewicz T., Wolski S., Jeżowski A. and Plackowski T., Appl. Phys. Lett., 89 (2006) 061901.

[15] Wetzel C., Wernicke T., Zeimer U., Brunner F., Weyers M. and Kneissl M., J. Cryst. Growth, 310 (2008) 8.

[16] Jiang W., Weber W. J. and Thevuthasan S., J. Appl. Phys., 87 (2000) 7671.

[17] Lorenz K., Wahl U., Alves E., Wojtowicz T., Ruterana P., Ruffenach S. and Briot O., Superlattices Microstruct., 36 (2004) 737.

[18] Ruterana P., Lacroix B. and Lorenz K., J. Appl. Phys., 109 (2011) 013506.

[19] Ziegler F., Biersack J. P. and Littmark U., The Stopping and Range of Ions in Solids (Pergamon, New York) 1985.

[20] Wendler E., Wesch W., Alves E. and Kamarou A., Nucl. Instrum. Methods Phys. Res. B, 218 (2004) 36. 
[21] Xiao H. Y., Gao F., Zu X. T. and Weber W. J., J. Appl. Phys., 105 (2009) 123527.

[22] Usov I. O., Arendt P. N., Groves J. R., Stan L. and DePaula R., Nucl. Instrum. Methods Phys. Res. B, 240 (2005) 661.

[23] Lacroix B., Leclerc S., Declémy A., Lorenz K., Alves E. and Ruterana P., EPL, 96 (2011) 46002.

[24] Liu C., Mensching B., Volz K. and Rauschenbach B., Appl. Phys. Lett., 71 (1997) 2313.
[25] Lorenz K., Peres M., Franco N., Marques J. G., Miranda S. M. C., Magalhães S., Monteiro T., Wesch W., Alves E. and Wendler E., Proc. SPIE, 7940 (2011) 79400 O.

[26] Geruschke T., Vianden R. and Lorenz K., unpublished.

[27] Hao R., Zhu T., Häberlen M., Chang T. Y., Kappers M. J., Oliver R. A., Humphreys C. J. and Moram M. A., J. Cryst. Growth, 312 (2010) 3536. 